Published in final edited form as:

J Arthroplasty. 2018 July ; 33(7): 2146-2152.e4. doi:10.1016/j.arth.2018.02.026.

\title{
Evaluating patients' expectations from a novel patient-centered perspective predicts knee arthroplasty outcome
}

\author{
Stephanie R Filbay, B.Phty(Hons), PhD, \\ Arthritis Research UK Centre for Sport, Exercise and Osteoarthritis, Nuffield, Department of \\ Orthopaedics, Rheumatology \& Musculoskeletal Sciences, University of Oxford, Botnar Research \\ Centre, Windmill Rd, Oxford UK OX3 7LD \\ Andrew Judge, PhD, \\ Department of Translational Health Sciences, Bristol Medical School, University of Bristol, \\ Musculoskeletal Research Unit, University of Bristol, School of Clinical Sciences, Bristol BS10 \\ 5NB, UK; Nuffield Department of Orthopaedics, Rheumatology and Musculoskeletal Sciences, \\ University of Oxford, Windmill Road, Oxford OX3 7LD, UK; MRC Lifecourse Southampton \\ General Hospital University of Southampton, Tremona Road, Southampton SO16 6YD, UK
}

Antonella Delmestri, PhD,

Nuffield Department of Orthopaedics, Rheumatology \& Musculoskeletal Sciences, University of Oxford, Botnar Research Centre, Windmill Rd, Oxford UK OX3 7LD

Nigel K Arden, MBBS, FRCP, MSc, MD, and

Arthritis Research UK Centre for Sport, Exercise and Osteoarthritis, Nuffield Department of Orthopaedics, Rheumatology \& Musculoskeletal Sciences, University of Oxford, Botnar Research Centre, Windmill Rd, Oxford UK OX3 7LD

on behalf of the COASt Study Group *

\section{Abstract}

Background-One in five patients are dissatisfied following knee arthroplasty and $<50 \%$ have fulfilled expectations. The relationship between knee-arthroplasty expectations and surgical outcome remains unclear.

Purpose-Are expectations regarding the impact of pain on life after knee arthroplasty predictive of one-year outcome? Does the impact of pain on preoperative quality of life (QOL) influence this relationship?

Methods-Longitudinal cohort study of 1044 uni-compartmental (43\%) or total kneearthroplasty (57\%) (UKA or TKA) patients, aged mean $69 \pm 9$ years. Preoperatively, patients reported the impact of pain on QOL and the expected impact of pain on life one-year postarthroplasty (none, mild, moderate/severe/extreme). One-year postoperative outcomes: non-return to desired activity (specific activities were specified preoperatively), surgical dissatisfaction, not achieving Oxford Knee Score (OKS) minimal important change (MIC). Logistic regression

corresponding author: stephanie.filbay@uq.net.au, Phone: 01865737254.

${ }_{*}^{*}$ Altman D, Beard D, Carr A, Cooper C, Culliford D, Griffin T, Javaid K, Latham J, Murray D, Pinedo-Villanueva R, Price A, PrietoAlhambra $D$. 
including covariates was performed for all patients and subgroups (better vs. worse pre-operative pain-related QOL; UKA vs. TKA; osteoarthritis indication vs. other indication).

Results-Expecting moderate-to-extreme pain (vs. no pain) predicted non-return to activity (odds ratio (95\% CI), 2.3(1.3, 4.1)), dissatisfaction (4.0(1.7, 9.3)), and not achieving OKS MIC $(3.1(1.5,6.3))$.

Expecting mild pain (vs. no pain) predicted worse outcomes for patients with better preoperative pain-related QOL (non-return to activity: 2.7(1.5, 4.8), not achieving OKS MIC: 2.5(1.1, 5.5)). Expecting moderate-to-extreme pain (vs. no pain) predicted worse outcomes for patients with worse preoperative pain-related QOL (non-return to activity: 2.4(1.1, 5.5), dissatisfaction: 5.0(1.7, 14.8), not achieving OKS MIC: $3.4(1.4,8.6))$.

The odds of a poor outcome in people with worse expectations was higher for UKA patients.

Conclusions-Expecting a worse outcome predicted surgical dissatisfaction, less clinical improvement and non-return to desired activity. Patients expecting a more optimistic outcome relative to preoperative status achieved better surgical outcomes.

\section{Keywords}

Quality of life; pain; satisfaction; patient-reported outcomes; optimism

\section{Introduction}

\section{Background and rationale}

Although knee arthroplasty surgery is considered an effective treatment for end-stage knee osteoarthritis [1,2], around 1 in 5 patients are dissatisfied with surgical outcome [3-5] and as many as $44 \%$ of patients experience persistent knee pain 3 to 4 years after surgery [6]. Knee arthroplasty patients commonly hold high expectations of surgery, with the majority expecting to be pain free with few functional limitations [7]. Despite high expectations, less than half of all patients will have these expectations fulfilled [7, 8]. However, the relationship between knee arthroplasty expectations and postoperative outcomes and satisfaction remains unclear with disagreement in recent literature [4, 9-11]. Potential explanations for conflicting findings include few studies controlling for confounding factors and a focus on expected physical outcomes without putting expectations within the context of an individual's quality of life (QOL) $[9,12,13]$. Pain is the most common reason patients choose to undergo joint arthroplasty [14] and pain expectations have been assessed by evaluating expected postoperative pain severity [11]. However, patients who experience the same severity of pain, may report contrasting impacts upon their QOL. Assessing the expected impact of pain on life after knee arthroplasty may be more meaningful to the patient and could provide new insights into the relationship between expectations and arthroplasty outcomes.

Several studies have reported an association between better preoperative status (including less knee pain, fewer knee limitations and better mental and general health) and high expectations of knee arthroplasty [13]. Since 80-90\% of patients have high expectations for surgery [7] there is likely to be a subgroup of patients with worse preoperative status who 
have unrealistically high expectations (e.g. to be pain-free with no functional impairment 12 months after surgery [7]). It is unclear if unrealistic expectations are associated with more dissatisfaction or worse outcome after knee arthroplasty. Although multiple studies exploring knee arthroplasty expectations have concluded by recommending promoting 'more realistic' patient expectations [3, 7, 8, 15], this may not be supported by current evidence. Optimistic expectations have been associated with greater improvement in pain and function 6-12 months after knee arthroplasty [4, 15-17]. Additionally, interventions inducing positive pain expectations and optimism result in pain relief and reduced pain sensitivity [11,18-20]. Considering preoperative education can shape knee arthroplasty expectations [21,22], there is potential to modify outcome by altering expectations. Further research exploring the relationship between preoperative status, surgical expectation and knee arthroplasty outcome is warranted.

This study will explore the relationship between expectations and knee arthroplasty outcomes from a novel, patient-centered perspective to provide clarity, new insights and build upon previous literature in this field. We aimed to answer the following questions:

(1) Are expectations regarding the impact of pain on life after knee arthroplasty predictive of one-year outcome?

(2) Does the impact of pain on preoperative QOL influence the relationship between expectation and one-year postoperative outcome?

\section{Materials and methods}

\section{Study design and setting}

The Clinical Outcomes in Arthroplasty Study (COASt) is a prospective, dual-centre longitudinal cohort study of patients undergoing hip and knee arthroplasty procedures at two UK hospitals. The overall aim of COASt was to produce a clinical tool for predicting the outcome of hip and knee arthroplasty including patient-reported outcomes, arthroplasty infection and long term prosthesis survival. A range of outcomes were collected at baseline (preoperative), six weeks and one year after knee and hip arthroplasty with annual follow-up planned for five years. This study has been approved by the Oxford REC A (Ethics Reference: 10/H0604/91).

\section{Participants}

Patients were recruited into COASt from the waiting lists for hip or knee arthroplasty at the Nuffield Orthopaedic Centre in Oxford UK (from 2010 to 2014), and the orthopaedic department at Southampton University Hospital NHS Foundation Trust in Southampton, UK (from 2010 to 2016). All patients on the waiting list were considered for the study. To be eligible for participation in COASt, individuals had to be aged over 18 years; on the waiting list for hip or knee arthroplasty; not have a severe neurological disorder; and be competent and willing to consent to partake in the study. For the current study, only individuals undergoing uni-compartmental knee arthroplasty (UKA) or primary total knee arthroplasty (TKA) were included in analyses, resulting in the exclusion of individuals undergoing hip procedures, revision TKA and patellofemoral procedures. 
Potentially eligible patients received a recruitment pack including a recruitment letter, study information sheet and consent form and were contacted approximately two weeks later to determine their eligibility and desire to take part in the study. If they verbally consented to partake in the study, a research appointment was arranged to undertake baseline assessment. At this appointment, written consent was obtained prior to completing preoperative questionnaires and a physical examination performed by a research nurse, physiotherapist or podiatrist which included assessment of strength, range of motion, function, musculoskeletal alignment, a whole body density (DXA) scan and collection of blood and urine samples. Participants were invited to complete a one-year follow-up questionnaire which was completed by post or on-site during a one-year follow-up appointment.

In total, 1801 patients who were on the waiting list for a knee arthroplasty gave initial verbal consent to take part in the study. 30 patients were excluded due to withdrawing consent $(n=6)$ or having a severe neurological disorder $(n=24) .1771$ eligible patients underwent baseline assessment. Between baseline assessment and undergoing knee arthroplasty, a further 257 patients were excluded from the study (Figure 1). Of the 1514 UKA and primary TKA procedures that were recorded, 36 patients died before one-year follow-up, 372 did not complete 1-year follow-up and 38 only completed follow-up over the telephone.

Additionally, 24 participants had undergone a contralateral UKA or primary TKA, since a second surgery could influence patient expectations, only data pertaining to their first procedure were included in this study. The sample size available for analysis, the retention rate and reasons for exclusion are depicted in Figure 1. In total, 1044 patients (74\%) who underwent a UKA or primary TKA and were eligible to complete one-year follow-up, participated in one-year follow-up. Indications for knee arthroplasty included osteoarthritis $(\mathrm{n}=848)$, rheumatoid arthritis $(\mathrm{n}=26)$, other inflammatory arthroplasty $(\mathrm{n}=12)$, avascular necrosis $(\mathrm{n}=8)$ and previous trauma $(\mathrm{n}=5)$.

\section{Patient reported outcomes}

The baseline and one-year follow-up questionnaires collected detailed demographic information and included a number of validated patient-reported outcomes. Information regarding preoperative expectations, return to desired activities and postoperative satisfaction with surgical outcome were also collected. Three one-year outcomes were chosen in line with the aims of this study, non-return to desired activity, dissatisfaction with surgical outcome and not achieving minimal important change (MIC) for the OKS.

Non-return to desired activity-At baseline, participants were asked 'What activity does your knee stop or limit you from doing that you wish to return to after your operation?' At one-year following knee arthroplasty, participants were asked: 'Have you been able to return to the activity (or activities) that your knee stopped you from doing one year ago? (yes/no).'

Dissatisfaction with surgical outcome-Participants responded to the following question at one-year follow-up: 'we would like to know your overall satisfaction with the outcome of your operation, please do this by ticking the option which best indicates your satisfaction' on a 5-point Likert scale (very satisfied, somewhat satisfied, neither satisfied 
nor dissatisfied, somewhat dissatisfied, very dissatisfied). Since a small proportion of participants selected the 'neither satisfied nor dissatisfied' response $(n=34,4 \%)$ participants selecting this response were removed from analysis to allow for dichotomisation into 'satisfied (very satisfied or somewhat satisfied)' and 'dissatisfied (somewhat dissatisfied or very dissatisfied)'.

Not achieving OKS MIC-The OKS was designed to assess the knee-related health status of patients undergoing TKA and comprises 12 items addressing knee pain and function [23]. The OKS has adequate test-retest reliability for use with individuals and groups, demonstrates good sensitivity, is responsiveness to change and is valid for use in TKA populations [24]. Items on the OKS are assigned a value from 4 (best) to 0 (worst), and items are summed to produce a total score on a scale from 0 to 48 , where a lower score represents more clinical impairment. In line with recommended guidelines, where one or two items were unanswered, the mean value of all other items was used to estimate missing values, where three or more items were unanswered, an overall index score was not given [25].

Baseline and one-year OKS values were used to calculate an OKS change-score by subtracting the baseline OKS from the one-year OKS for each participant. The MIC for the OKS following TKA at an individual level, has been estimated to be 7-points (considered as the minimal amount of change necessary to distinguish between patients 'a little better' from those 'about the same' in a UK sample of 94,502 individuals undergoing knee arthroplasty) [26]. To enhance interpretation of findings and maintain consistency with other binary outcomes, the OKS was dichotomised using a cut-off of 7-points, whereby patients reporting an improvement of 6-points or less on the OKS between baseline and one-year follow-up were categorised as 'not achieving OKS MIC'.

\section{Predictor variable}

Expected impact of pain on life one-year after arthroplasty-Participants were asked to respond to the following question at baseline: 'Overall, how much do you expect that pain in your knee will interfere with your life one year after surgery?' on a five-point Likert scale (not at all, mildly, moderately, severely, extremely). Due to few participants expecting a severe or extreme impact of pain on life post-arthroplasty $(n=6,1 \%)$, this variable was re-coded into 3 categories for analyses: 'none' vs. 'mild' vs. 'moderate-toextreme.'

\section{Covariates}

Covariates were selected based upon clinical relevance and literature review to identify factors associated with postoperative outcome after knee arthroplasty. The following covariates were identified for inclusion in multivariable models: gender, age at operation, education level (higher education vs. other), current smoker (yes/no), body mass index (BMI), number of co-morbidities (co-morbidities assessed: high cholesterol, osteoporosis, gout, renal problems, bowel problems, lung problems, diabetes, liver problems, heart failure, heart attack, stroke, hypertension), surgery type (primary TKA vs. UKA) and baseline EQ-5D score (index values were calculated using value sets from the United Kingdom [27]). 


\section{Statistical analysis}

Multiple imputation using 40 iterations was performed to account for missing values (Table 1) using the Markov Chain Monte Carlo (MCMC) technique [28]. Separate imputation models were performed for each of the three different outcomes. Underlying assumptions for multiple imputation were assessed and distributions, descriptives and odds ratios were compared between raw and imputed data sets to ensure the imputed dataset accurately reflected the raw data.

Binary logistic regression was performed to investigate the relationship between preoperative expectations and one-year outcome. All underlying assumptions for logistic regression analysis were assessed and satisfied prior to conducting the analyses (including linearity and multicollinearity of independent variables, investigation of outliers and distribution of residuals). Uni-variable (crude) and multi-variable (adjusted) analyses were performed and odds ratios (95\% CIs) reported. All potential covariates identified through clinical reasoning and literature review were included in the multivariable models. Interaction effects were assessed for type of procedure (UKA vs. TKA) and expectations; and found to be non-significant for all outcomes. To minimise the likelihood of effect estimate inflation and to aid in clinical interpretation, the rarer outcomes (dissatisfaction, non-return to desired activity and not achieving MIC for the OKS) were chosen as reference categories (coded as 1) in multivariable analyses.

To address the second aim of this study, patients were stratified into one of two subgroups based on the following question 'How much does pain in your knee affect your overall QOL now?' 'none/mild/moderate' ('better preoperative pain-related QOL') vs. 'severe/extreme' ('worse preoperative pain-related QOL'). A subgroup analysis was performed using the same variables and procedure as the main analysis. Since this was an exploratory subgroup analysis it was performed on non-imputed raw data.

Additionally, two exploratory subgroup analyses were performed; the first stratified patients by knee arthroplasty procedure (UKA vs. TKA), and the second analysis stratified patients by knee arthroplasty indication (knee osteoarthritis vs. all other indications). The results of these subgroup analyses will be summarised in-text and presented in full in Supplementary Appendices. All analyses and multiple imputation was performed using Stata/IC 14.1

\section{Results}

\section{Participant characteristics}

Participants were aged a mean 69 SD 9 years at the time of surgery (range 28 to 90 years) and $57 \%$ underwent primary TKA $(\mathrm{n}=597)$, as opposed to UKA $(\mathrm{n}=447) .576(55 \%)$ of participants were female, $84 \%$ had $\geq 1$ comorbidity $(n=805)$ and 1 in 5 had $\geq 4$ comorbidities. Most participants reported a moderate $(n=262,36 \%)$ or severe $(n=318,43 \%)$ impact of pain on preoperative QOL. Most participants expected pain to have no impact $(\mathrm{n}=406,54 \%)$ or only a mild impact $(\mathrm{n}=285,38 \%)$ on life one-year after surgery, compared with a moderate $(n=53,7 \%)$, severe $(n=5,1 \%)$ or extreme $(n=1,0.5 \%)$ impact. Participant characteristics and one-year outcomes are presented in Table 1 for all participants, and stratified by knee arthroplasty procedure and preoperative expectation. 
Are expectations regarding the impact of pain on life after knee arthroplasty predictive of one year outcome?

Patients who expected mild pain after surgery had a 1.6 times greater odds of not returning to their desired activity compared to those who expected no pain (Table 2). Patients who expected moderate-to-extreme pain (compared to no pain) had a 2.3 times greater odds of not returning to their desired activity, a 4 times greater odds of being dissatisfied with surgical outcome, and a 3.1 times greater odds of not meeting the MIC of 7-points on the OKS (Table 2).

Surgical procedure and surgery indication-Worse expectations (mild or moderateto-extreme) was associated with not returning to desired activity following UKA but not following TKA (Supplementary Appendix 1). The relationship between expectation and other outcomes were similar following UKA and TKA, although odds ratios were higher for each outcome following UKA (Supplementary Appendix 1). Stratifying the sample by patients who underwent knee arthroplasty due to knee osteoarthritis compared with all other indications did not alter the study findings (Supplementary Appendix 2).

\section{Does the impact of pain on preoperative QOL influence this relationship?}

Patients who reported better preoperative pain-related QOL had a 2.7 times greater odds of not returning to desired activity and a 2.5 times greater odds of not achieving OKS MIC if they expected mild pain (compared to no pain) (Table 3). Expecting mild pain (compared to no pain) was not related to postoperative outcomes for patients who reported worse preoperative pain-related QOL.

Patients who reported worse preoperative pain-related QOL who expected moderate-toextreme pain (compared to no pain) had a 2.4 times greater odds of not returning to desired activity, a 5 times greater odds of being dissatisfied with surgery, and a 3.4 times greater odds of not achieving OKS MIC (Table 3).

Surgical procedure and surgery indication-Reporting better preoperative painrelated QOL and expecting mild pain (compared to no pain) was associated with not returning to desired activity following UKA (6.4 (2.1 to 19.8$)$ ) but not TKA (1.8 (0.8 to 3.7)). Reporting worse preoperative pain-related QOL and expecting moderate-to-extreme pain (compared to no pain) was associated with not achieving OKS MIC following UKA (6.6 (1.5 to 29.9)) but not TKA (2.9 (0.8 to 10.2)). Other results were similar following UKA and TKA procedures (Supplementary Appendix 1). Study findings did not change when the analyses were repeated in the subgroup of patients who underwent joint replacement due to knee osteoarthritis (Supplementary Appendix 2).

\section{Discussion}

There is a need to identify modifiable risk factors of poor outcome following knee arthroplasty. Expectations are modifiable through education, yet it is not clear if promoting realistic expectations is in a patient's best interest. Additionally, few studies have considered 
expectations in the context of a patient's QOL which may partly explain conflicting findings in previous studies investigating knee arthroplasty expectations and surgical satisfaction.

We found that expecting a greater impact of pain on life one year after knee arthroplasty, predicted surgical dissatisfaction, less clinical improvement and non-return to desired activity one-year following surgery. A recent systematic review found that greater expectations of knee arthroplasty surgery and expectation fulfilment were associated with better postoperative outcomes [11]. However, another review including a wider scope of studies found no relationship between patient expectations of knee arthroplasty and postoperative satisfaction or clinical improvement, as well as inconsistent associations between expectations and postoperative pain and function [11]. These inconsistencies may be partly explained by study design (including low sample size and not adjusting for confounding) and not assessing expectations of importance and relevance to the individual. Patients may be better able to predict the impact of pain upon their life as opposed to the severity of pain, which may be more influenced by external factors. Assessing expectations within the context of a patient's life may be more closely related to patient-centred outcomes, such as dissatisfaction and return to desired activities, compared with assessing expected pain severity, symptoms or function which may not align with a patient's life priorities.

Our subgroup analysis found that expecting a mild pain impact (as opposed to no impact) on life after arthroplasty only predicted worse outcome in patients reporting better preoperative pain-related QOL. Expecting a mild pain impact on life after arthroplasty could be considered a positive expectation for individuals who report more severe preoperative painrelated QOL impairment. In our study, preoperative EQ-5D scores tended to be more impaired and BMI tended to be higher, in patients who expected a worse outcome (Table 1). Despite this, adjusting for these baseline variables did not alter the positive relationship between expectations and outcome. This is in agreement with the broader medical literature, where controlling for the effect of confounding factors including psychological and social variables has little effect on the relationship between expectations and outcome [29]. These findings suggest that expectations may independently influence outcomes following knee arthroplasty. Considering a patients' expectations relative to the impact of their knee on their pre-operative QOL may provide useful information for identifying individuals at risk of experiencing unsatisfactory surgical outcomes.

The odds of a poor surgical outcome in people with worse expectations was higher for UKA patients. UKA patients tended to be younger (mean age 67 vs. 70), with a better baseline EQ-5D score (mean 0.47 vs. 0.44 ) and less comorbidities (no comorbidities, $22 \%$ vs. $12 \%$ ) than those who underwent TKA. Additionally, a higher proportion of UKA patients returned to their desired activity after surgery ( $75 \%$ vs. $59 \%$ ). There may have been other differences in patient characteristics between UKA and TKA patients that were not accounted for in our analyses (such as resilience, locus of control, fear-avoidance behaviour, social support) that may have influenced the relationship between expectations and surgical outcome. It is also possible that the type of activity patients wanted to return to differed between patients undergoing UKA compared with TKA patients. Further research is needed to explore differences in the relationship between expectation and outcome in UKA and TKA patients. 
Looking more broadly than joint arthroplasty surgery, positive surgical expectations have been associated with greater perceived improvement after surgery despite no change in objective measures of physical symptoms or health [30]. Positive expectations may signify greater levels of optimism, which in turn could have a positive effect on actual and/or perceived surgical outcome. Surgical placebo studies provide further insight into the way in which preoperative expectations may shape postoperative outcome. Since no active intervention is given during a placebo treatment, the impact of the placebo may be perceived in part, as a measure of the impact of patient expectation and beliefs. Beliefs in expectation and optimism have been identified as predictors of a positive placebo response [31]. Akin to a placebo treatment inducing a physiological response, an individual's perceptions and expectations can impact disease processes and pain severity [11, 18-20, 32].

Furthermore, expecting a favourable outcome can result in renewed effort, greater motivation, persistence and focus, in contrast to expecting an unfavourable outcome, which may result in decreased effort and disengagement [30,33]. It is possible that patients with greater outcome expectations were more engaged in postoperative rehabilitation and executed greater persistence in working toward their surgical goals, increasing the likelihood of that expectancy being fulfilled. Further research is needed to improve current understanding of the mechanisms by which preoperative expectations effect postoperative outcomes, such information could provide new targets for preoperative interventions to optimise postoperative outcomes.

\section{Strengths and limitations}

Due to the nature of study recruitment and the comprehensive study questionnaire, there was missing data for some measures. This was accounted for using multiple imputation. We did not impute the one-year outcomes resulting in a different sample of responders for each of the three outcomes. Although we accounted for total number of co-morbidities in analyses, we did not take into consideration the severity of each comorbidity or the varying impact of each on surgical outcome. We also recognise that using MIC to define important change for the OKS has limitations. A study in TKA patients found that attaining an acceptable level of function after surgery was more common than a clinically important improvement on a patient-reported measure [34]. Thus, a proportion of participants may have been satisfied with knee improvement despite reporting less than 7-point improvement on the OKS. However, this limitation was offset by including a combination of patient-centred (dissatisfaction and return to desired activity) and objective (OKS) measures, providing a holistic view of patient outcome. Other strengths include assessing expectations within the context of an individual's QOL, the large sample size and adjustment for a variety of covariates, which were common limitations in previous research.

\section{Conclusions}

In summary, patients who expected an optimistic surgical outcome were more likely to do better after surgery, irrespective of preoperative knee status. The association between worse expectations and worse outcomes was strongest following UKA (compared with TKA). Expecting pain to mildly impact postoperative life (as opposed to no impact) was only 
predictive of worse outcome in patients reporting better preoperative pain-related QOL. This suggests expectations should be interpreted relative to the impact of a patient's knee upon preoperative QOL. Evaluating pain expectations within the context of a patient's QOL may be meaningful to the patient and assist with identifying individuals at risk of unsatisfactory postoperative outcome.

\section{Abbreviations}

$\begin{array}{ll}\text { QOL } & \text { quality of life } \\ \text { OKS } & \text { Oxford Knee Score } \\ \text { MIC } & \text { minimal important change } \\ \text { COASt } & \text { Clinical Outcomes in Arthroplasty Study } \\ \text { UK } & \text { United Kingdom } \\ \text { UKA } & \text { uni-compartmental knee arthroplasty } \\ \text { TKA } & \text { total knee arthroplasty } \\ \text { BMI } & \text { body mass index }\end{array}$

\section{References}

1. Losina E, Walensky RP, Kessler CL, Emrani PS, Reichmann WM, Wright EA, Holt HL, Solomon DH, Yelin E, Paltiel AD, Katz JN. Cost-effectiveness of total knee arthroplasty in the United States: patient risk and hospital volume. Arch Intern Med. 2009; 169

2. Quintana JM, Escobar A, Arostegui I, Bilbao A, Azkarate J, Goenaga JI, Arenaza JC. Health-related quality of life and appropriateness of knee or hip joint replacement. Arch Intern Med. 2006; 166(2): 220. [PubMed: 16432092]

3. Nilsdotter AK, Toksvig-Larsen S, Roos EM. Knee arthroplasty: are patients' expectations fulfilled? A prospective study of pain and function in 102 patients with 5-year follow-up. Acta Orthop. 2009; 80

4. Gandhi R, Davey JR, Mahomed N. Patient expectations predict greater pain relief with joint arthroplasty. J Arthroplasty. 2009; 24(5):716. [PubMed: 18701241]

5. Scott CE, Bugler KE, Clement ND, MacDonald D, Howie CR, Biant LC. Patient expectations of arthroplasty of the hip and knee. J Bone Joint Surg Br. 2012; 94(7):974. [PubMed: 22733956]

6. Wylde V, Hewlett S, Learmonth ID, Dieppe P. Persistent pain after joint replacement: Prevalence, sensory qualities, and postoperative determinants. Pain. 2011; 152(3):566. [PubMed: 21239114]

7. Mannion AF, Kampfen S, Munzinger U, Kramers-de Quervain I. The role of patient expectations in predicting outcome after total knee arthroplasty. Arthritis research \& therapy. 2009; 11

8. Gonzalez Saenz de Tejada M, Escobar A, Herrera C, Garcia L, Aizpuru F, Sarasqueta C. Patient expectations and health-related quality of life outcomes following total joint replacement. Value Health. 2010; 13(4):447. [PubMed: 20088892]

9. Barlow T, Clark T, Dunbar M, Metcalfe A, Griffin D. The effect of expectation on satisfaction in total knee replacements: a systematic review. Springerplus. 2016; 5:167. [PubMed: 27026864]

10. Gonzalez Saenz de Tejada M, Escobar A, Bilbao A, Herrera-Espineira C, Garcia-Perez L, Aizpuru F, Sarasqueta C. A prospective study of the association of patient expectations with changes in health-related quality of life outcomes, following total joint replacement. BMC Musculoskelet Disord. 2014; 15:248. [PubMed: 25055728] 
11. Haanstra TM, Van Den Berg T, Ostelo RW, Poolman RW, Jansma IP, Cuijpers P, de Vet HCW. Systematic review: Do patient expectations influence treatment outcomes in total knee and total hip arthroplasty? Health and Quality of Life Outcomes. 2012; 10

12. Haanstra TM, van den Berg T, Ostelo RW, Poolman RW, Jansma IP, Cuijpers P, de Vet HC. Systematic review: Do patient expectations influence treatment outcomes in total knee and total hip arthroplasty? Health and Quality of Life Outcomes. 2012; 10(1):1. [PubMed: 22208808]

13. Dyck BA, Zywiel MG, Mahomed A, Gandhi R, Perruccio AV, Mahomed NN. Associations between patient expectations of joint arthroplasty surgery and pre- and post-operative clinical status. Expert Rev Med Devices. 2014; 11(4):403. [PubMed: 24793876]

14. Cremeans-Smith JK, Boarts JM, Greene K, Delahanty DL. Patients' reasons for electing to undergo total knee arthroplasty impact post-operative pain severity and range of motion. Journal of Behavioral Medicine. 2009; 32(3):223. [PubMed: 19137422]

15. Mahomed NN, Liang MH, Cook EF, Daltroy LH, Fortin PR, Fossel AH, Katz JN. The importance of patient expectations in predicting functional outcomes after total joint arthroplasty. $\mathrm{J}$ Rheumatol. 2002; 29

16. Lingard EA, Sledge CB, Learmonth ID. Patient expectations regarding total knee arthroplasty: differences among the United States, United kingdom, and Australia. The Journal of bone and joint surgery American. 2006; 88

17. Cross M, Lapsley H, Barcenilla A, Parker D, Coolican M, March L. Patient expectations of hip and knee joint replacement surgery and postoperative health status. The Patient. 2009; 2

18. Price DD, Finniss DG, Benedetti F. A comprehensive review of the placebo effect: recent advances and current thought. Annual review of psychology. 2008; 59:565.

19. Keltner JR, Furst A, Fan C, Redfern R, Inglis B, Fields HL. Isolating the modulatory effect of expectation on pain transmission: a functional magnetic resonance imaging study. J Neurosci. 2006; 26(16):4437. [PubMed: 16624963]

20. Hanssen MM, Peters ML, Vlaeyen JW, Meevissen YM, Vancleef LM. Optimism lowers pain: evidence of the causal status and underlying mechanisms. Pain. 2013; 154(1):53. [PubMed: 23084002]

21. Mancuso CA, Graziano S, Briskie LM, Peterson MGE, Pellicci PM, Salvati EA, Sculco TP. Randomized Trials to Modify Patients' Preoperative Expectations of Hip and Knee Arthroplasties. Clinical orthopaedics and related research. 2008; 466(2):424. [PubMed: 18196427]

22. McDonald S, Page MJ, Beringer K, Wasiak J, Sprowson A. Preoperative education for hip or knee replacement. Cochrane Database Syst Rev. 2014; 5 Cd003526.

23. Murray DW, Fitzpatrick R, Rogers K, Pandit H, Beard DJ, Carr AJ, Dawson J. The use of the Oxford hip and knee scores. Journal of Bone \& Joint Surgery, British. 2007; 89-B(8):1010.

24. Collins N, Misra D, Felson D, Crossley KM, Roos EM. Measures of knee function. Arthritis Care and Research. 2011; 63(11S):S1. [PubMed: 22588738]

25. Oxford University Innovation. Oxford Knee Score Scoring Guide. University of Oxford; 2007.

26. Beard DJ, Harris K, Dawson J, Doll H, Murray DW, Carr AJ, Price AJ. Meaningful changes for the Oxford hip and knee scores after joint replacement surgery. Journal of Clinical Epidemiology. 2015; 68(1):73. [PubMed: 25441700]

27. Ramos-Goni JM, Rivero-Arias O. eq5d: A command to calculate index values for the EQ-5D quality-of-life instrument. Stata Journal. 2011; 11(1):120.

28. Sterne JAC, White IR, Carlin JB, Spratt M, Royston P, Kenward MG, Wood AM, Carpenter JR. Multiple imputation for missing data in epidemiological and clinical research: potential and pitfalls. BMJ. 2009; 338

29. Mondloch MV, Cole DC, Frank JW. Does how you do depend on how you think you'll do? A systematic review of the evidence for a relation between patients' recovery expectations and health outcomes. CMAJ: Canadian Medical Association journal = journal de l'Association medicale canadienne. 2001; 165(2):174.

30. Flood AB, Lorence DP, Ding J, McPherson K, Black NA. The role of expectations in patients' reports of post-operative outcomes and improvement following therapy. Med Care. 1993; 31(11): 1043. [PubMed: 7694013] 
31. Horing B, Weimer K, Muth ER, Enck P. Prediction of placebo responses: A systematic review of the literature. Frontiers in Psychology. 2014 Oct.5

32. Cohen S, Herbert TB. Health psychology: psychological factors and physical disease from the perspective of human psychoneuroimmunology. Annual review of psychology. 1996; 47:113.

33. Scheier MF, Carver CS. Optimism, coping, and health: assessment and implications of generalized outcome expectancies. Health psychology : official journal of the Division of Health Psychology, American Psychological Association. 1985; 4(3):219.

34. Maxwell JL, Felson DT, Niu J, Wise B, Nevitt MC, Singh JA, Frey-Law L, Neogi T. Does clinically important change in function after knee replacement guarantee good absolute function? The multicenter osteoarthritis study. J Rheumatol. 2014; 41(1):60. [PubMed: 24293582] 


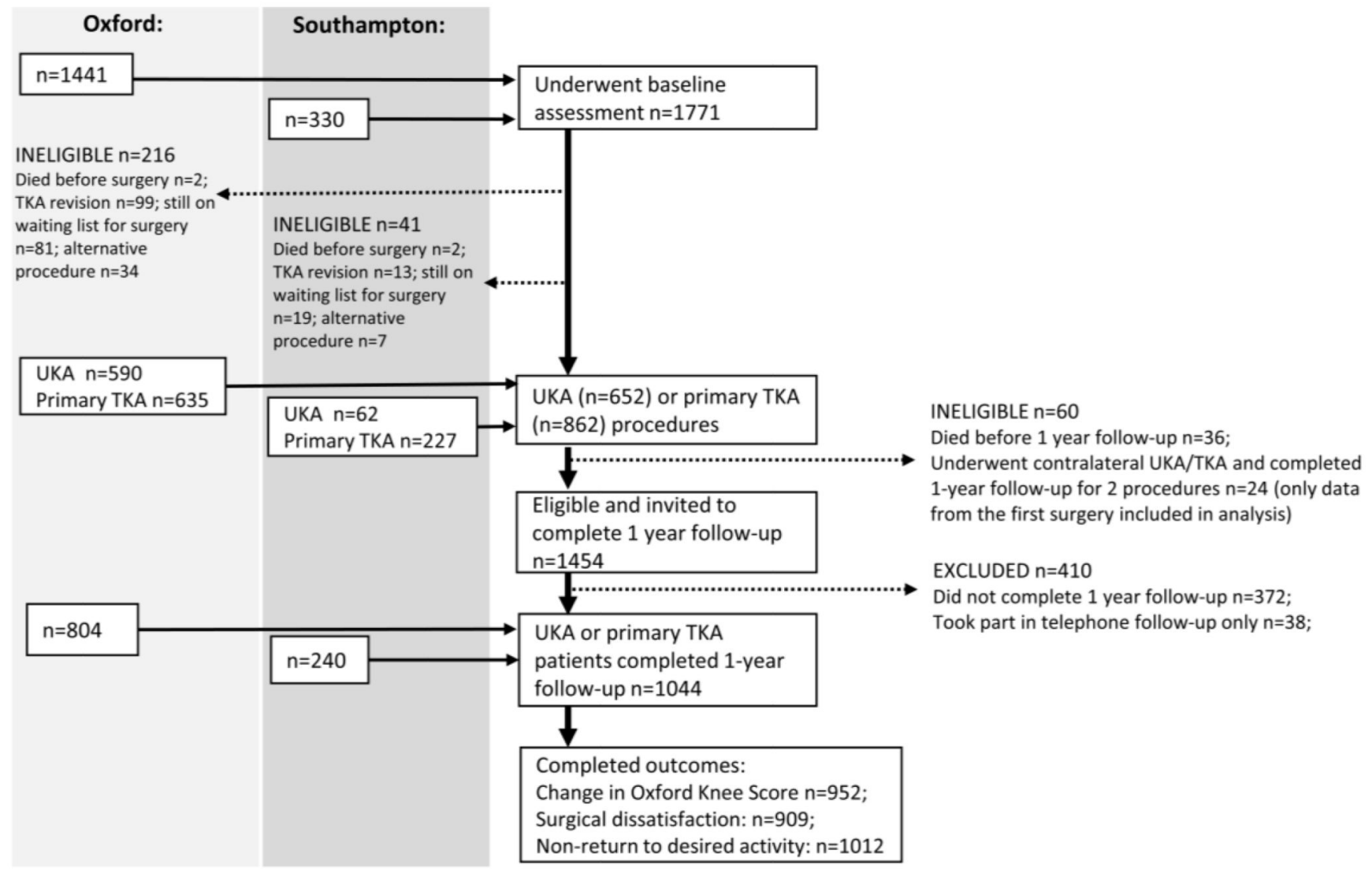

Figure 1. 


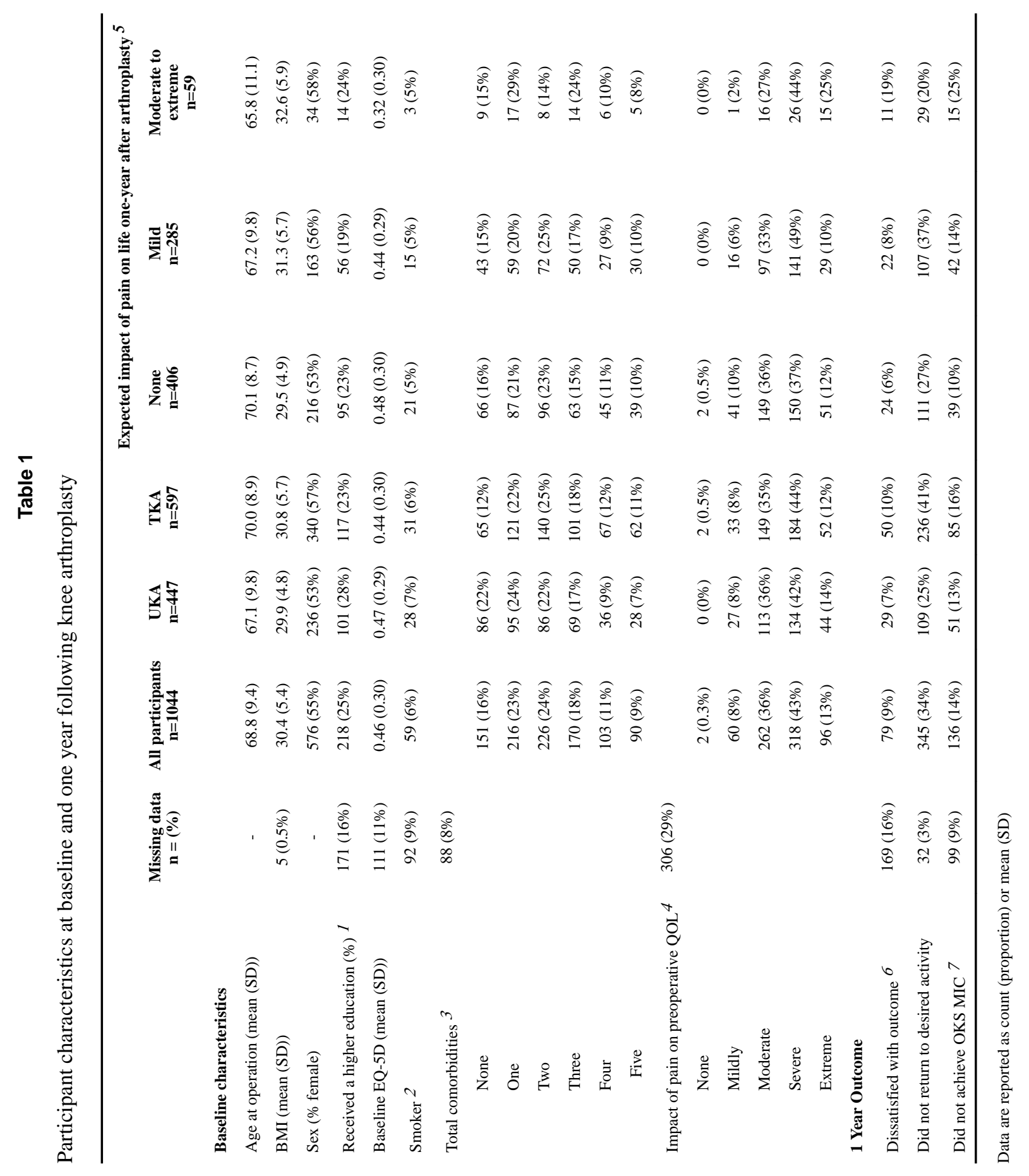




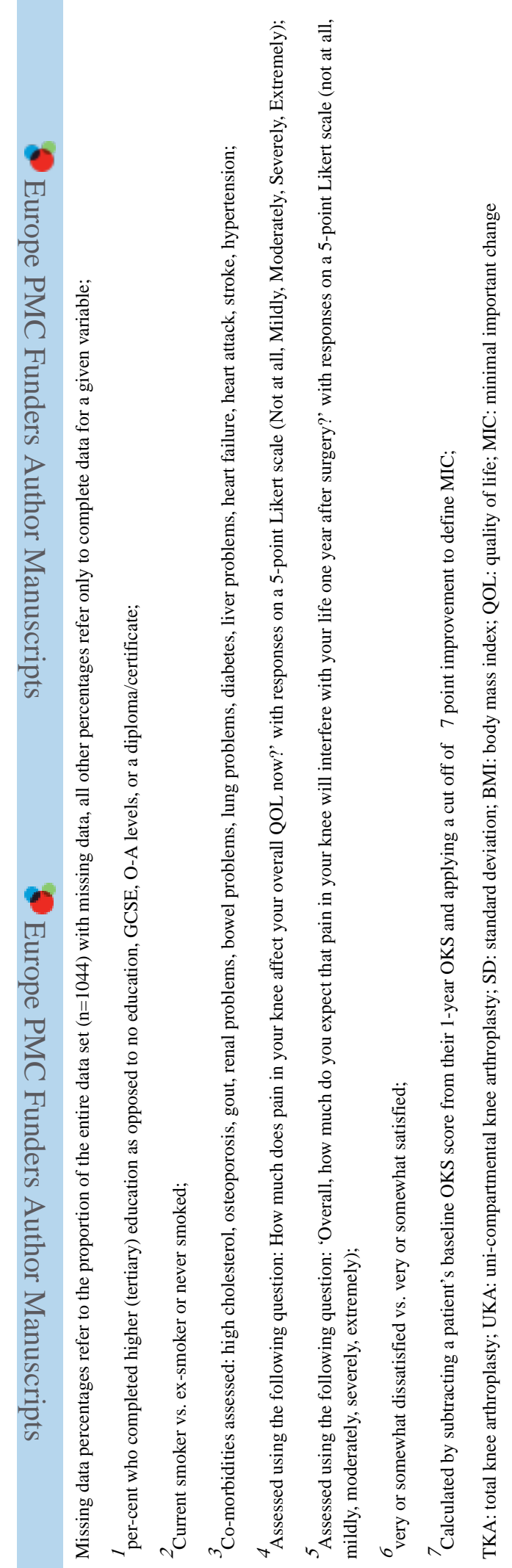

J Arthroplasty. Author manuscript; available in PMC 2019 January 01. 


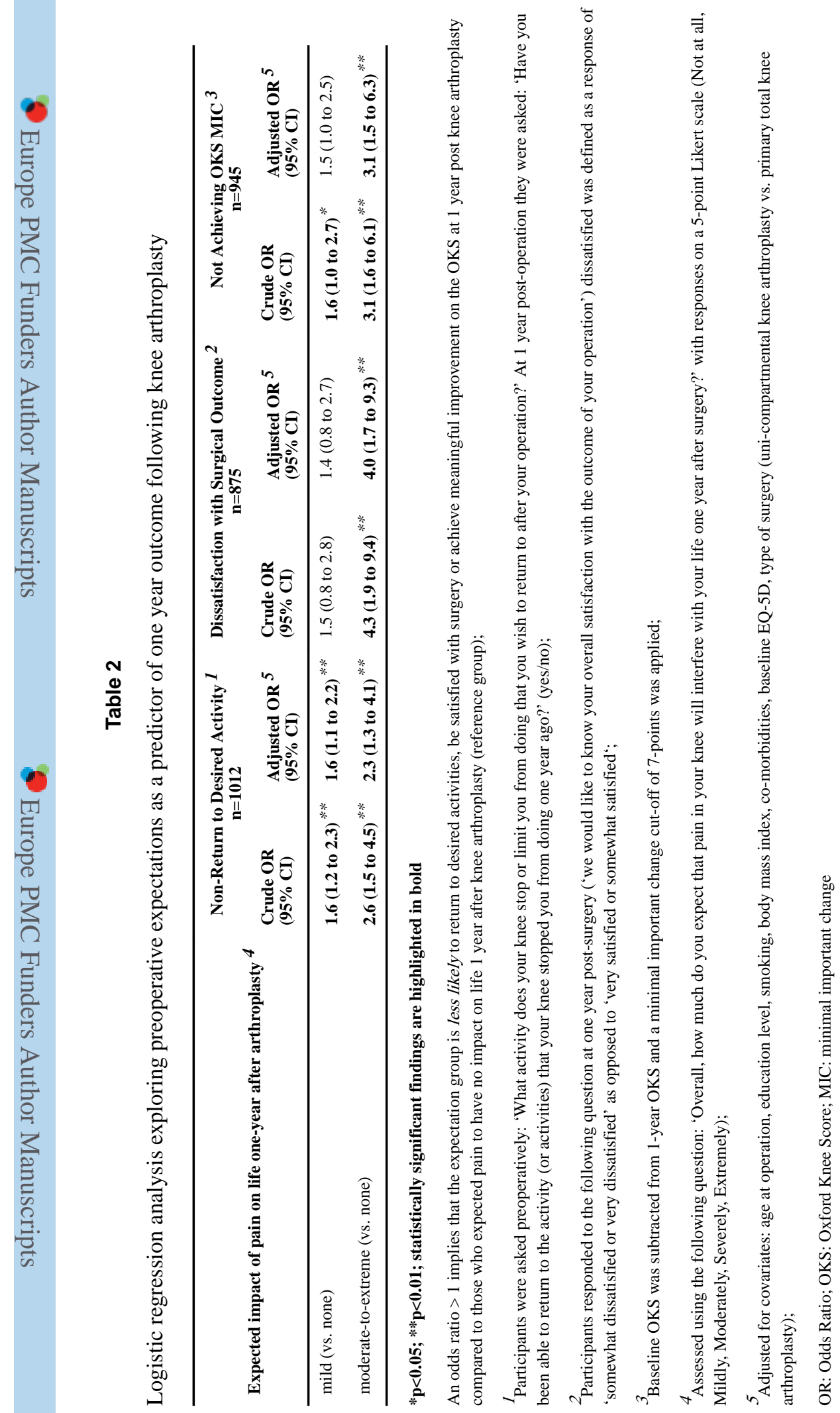

J Arthroplasty. Author manuscript; available in PMC 2019 January 01. 


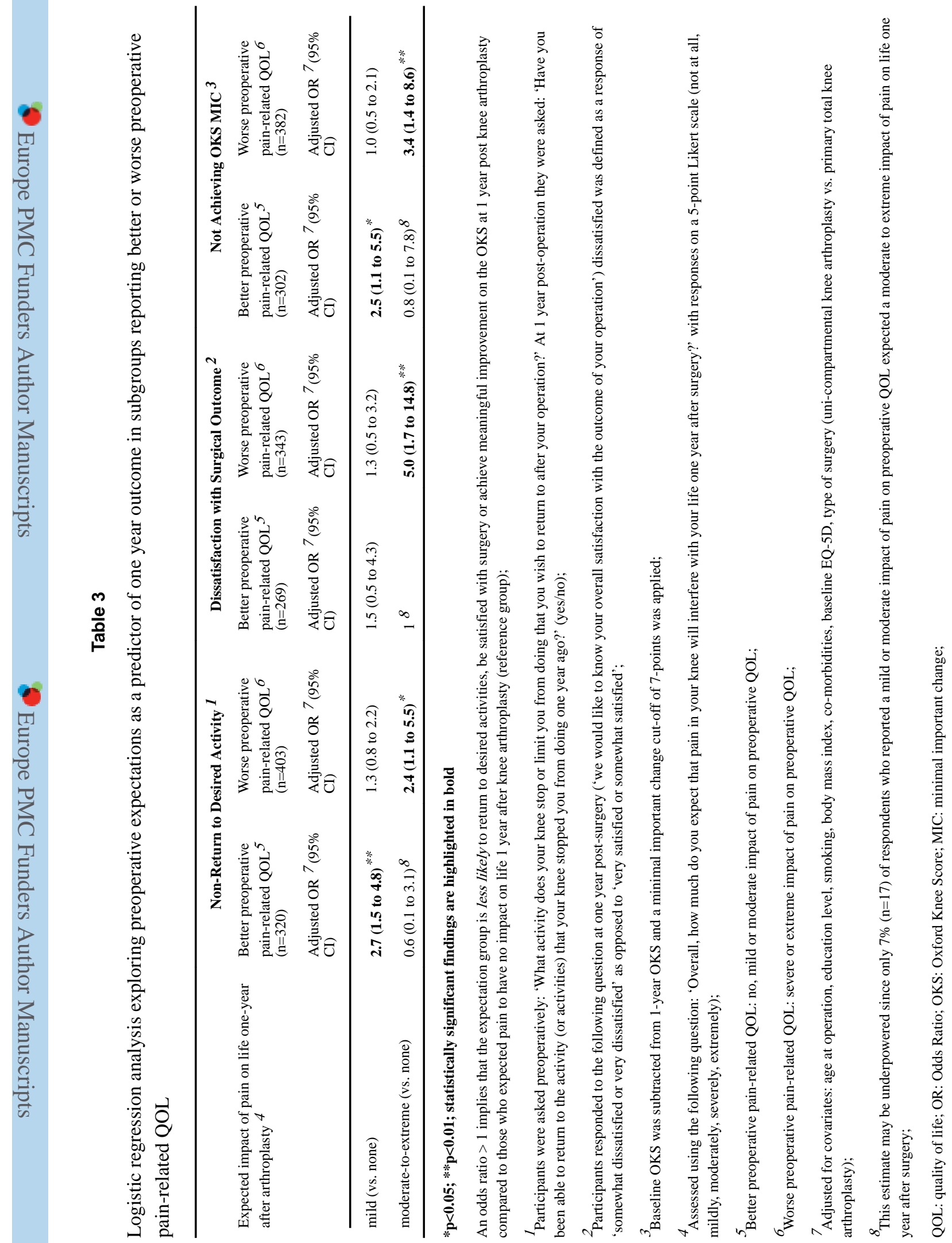

